\title{
PROJECTIVE TEXTURING UNCERTAIN GEOMETRY: SILHOUETTE-AWARE BOX-FILTERED BLENDING USING INTEGRAL RADIAL IMAGES
}

\author{
Mathieu Brédif \\ Université Paris-Est, IGN, SRIG, MATIS, 73 avenue de Paris, 94160 Saint Mandé, France \\ mathieu.bredif @ign.fr
}

KEY WORDS: Projective texturing, Uncertainty, Image based rendering

\begin{abstract}
:
Projective texturing is a commonly used image based rendering technique that enables the synthesis of novel views from the blended reprojection of nearby views on a coarse geometry proxy approximating the scene. When scene geometry is inexact, aliasing artefacts occur. This introduces disturbing artefacts in applications such as street-level immersive navigation in mobile mapping imagery, since a pixel-accurate modelling of the scene geometry and all its details is most of the time out of question. The filtered blending approach applies the necessary 1D low-pass filtering on the projective texture to trade out the aliasing artefacts at the cost of some radial blurring. This paper proposes extensions of the filtered blending approach. Firstly, we introduce Integral Radial Images that enable constant time radial box filtering and show how they can be used to apply box-filtered blending in constant time independently of the amount of depth uncertainty. Secondly, we show a very efficient application of filtered blending where the scene geometry is only given by a loose depth interval prior rather than an actual geometry proxy. Thirdly, we propose a silhouette-aware extension of the box-filtered blending that not only account for uncertain depth along the viewing ray but also for uncertain silhouettes that have to be blurred as well.
\end{abstract}

\section{INTRODUCTION}

\subsection{Context}

Many 3D visualization applications do not really require a precise and accurate modelling of the scene geometry and radiometry. What is really required in the end is the synthesis of a novel viewpoint with minimal temporal or spatial artefacts and the best quality possible. By bypassing the modelling step, that is what image-based rendering techniques are targeting (Brédif, 2013).

\subsection{Rendering uncertain geometry}

The geometry of the real world is only captured (stereo reconstruction, lidar sensing...) at a given resolution, up to a certain level of accuracy and with some amount of noise and outliers. We are interested in propagating this uncertainty of the geometry (point cloud, 3D model...) to the rendering to prevent arbitrary decision and information loss.

Hofsetz (2003) proposed an image based rendering technique that handles a point cloud with uncertainty. Each point is splat on the screen using its uncertainty ellipse to get the relevant filtering. This approach gives satisfactory results but scales not so well with the number of uncertain points.

The filtered-blending approach of Eisemann et al. (2007) is motivated by the fact that an incorrect geometric approximation of the scene will yield aliasing artefacts when colored by projecting multiple textures on it. They proved that only a 1D filtering was needed to prevent that aliasing. A second approach is to let the projective parameters of the texture evolve so as to reduce the visible artefacts. This is the floating texture approach of Eisemann et al. (2008).

The Ambient Point Cloud method (Goesele et al., 2010) has been developed in the context of multi view stereo where part of the scene is densely reconstructed, part of the scene is composed of a sparse point set and some pixels do not have any depth estimate, each with a different rendering mode in accordance to the different nature of geometric uncertainty. This paper introduced a notion similar to Eisemann et al. (2007) that uncertain depth may be rendered as a linear filtering in the epipolar motion direction. They however preferred a filtering based of sparse and jittered point samples rather than the low-pass filtering discussed in Eisemann et al. (2007).

\subsection{Contributions}

Focusing on box filters, we propose to extend the filtered-blending approach of Eisemann et al. (2007) in a number of aspects :

- We adapt the concept of constant time box filtering provided by $2 \mathrm{D}$-integral images to computing box-filtered blending with a performance which is independent of the blur extent, by introducing the integral radial images (sections 2 and 3.2).

- Application-wise, we show how this method may be straightforwardly adapted to projective-textured scenes where geometry is given only by a loose depth prior, which yields satisfactory results in the showcased street-level navigation context (section 3.1).

- Lastly, our main contribution is an extension of box-filtered blending that handles uncertain silhouette boundaries (section 3.3).

\section{CONSTANT-TIME BOX-FILTERED BLENDING}

\subsection{Epipolar Filtering}

The interpolated view to be synthesized by filtered blending and each projective texture view may be considered as a stereo rig, as they are both defined by conic projections. Thus, the 3D ray originating from a pixel location in the interpolated view projects in the (undistorted) projective texture image plane as a $2 \mathrm{D}$ ray with its origin at the epipole : the projection of the interpolated viewpoint on the projective texture. We will denote such a ray an epipolar ray. 


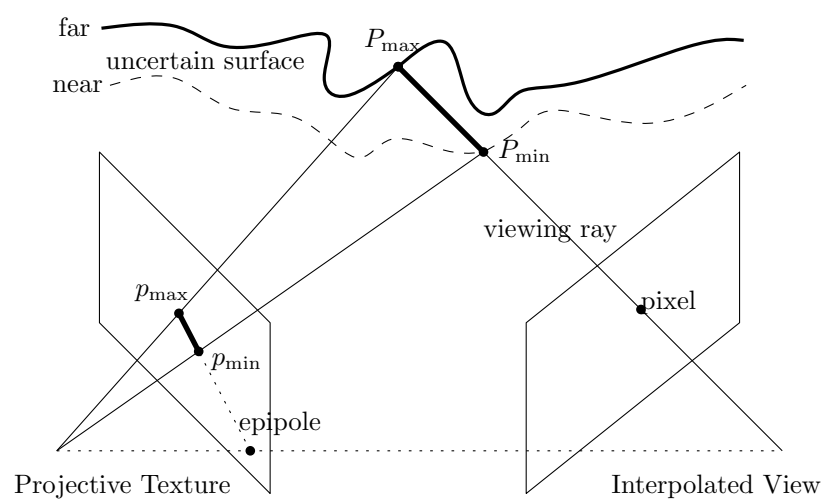

Figure 1: Epipolar filtering geometry

For a given pixel, scene uncertainty translates into an uncertainty of the 3D location (e.g. $\left[P_{\min }, P_{\max }\right]$ in figure 1) of the intersection of the $3 \mathrm{D}$ pixel ray with the scene, and consequently to an uncertain 2D position on the corresponding epipolar ray (e.g. $\left[p_{\min }, p_{\max }\right]$ in figure 1$)$. The kernel used in filtered blending is essentially defining, for each projective texture to be blended, a probability density of this $2 \mathrm{D}$ location on the epipolar ray in ordered to compute its expected value.

As all pixel rays will reproject to epipolar rays, the resulting expected values will all be integrated over linear supports that meet at the projective texture epipole. Thus, the filters used in the filtered blending of each projective texture reduce to $1 \mathrm{D}$ filters in a radial setup with a pole fixed at the epipole.

The most simple probability density is a uniform density over the subset of the $2 \mathrm{D}$ epipolar rays that correspond to the projection of plausible view ray / scene intersections. This uniform density translates into radial 1D-box filters around the epipole.

\subsection{Linear-Time Filtered Blending}

With box filtering, filtered blending requires computing average pixel values over line segments. For segments of small length, the marching along this line segment may be performed exhaustively by stepping through and sampling directly the original texture. However, for longer segments, the linear increase of samples (texture lookups) becomes a performance bottleneck. A quick and dirty solution is to limit the number of samples along the segment to a fixed maximum. This may be approximated and implemented in a number of ways including (figure 2) :

- No prefiltering. This generates, for long segments, a grainy look similar to what is achieved in the Ambient Point Cloud approach (Goesele et al., 2010), which may lead to aliasing or temporal incoherence due to the unfiltered point sampling of the input texture.

- Isotropic prefiltering. This approach aims to approximate the integral over the linear segment as a sum of samples on a blurred (i.e. band-limited) image. It may be easily implemented using mipmap level biasing in the graphics pipeline. This however introduces a trade off between the necessary unaliasing blur along the segment and the unnecessary blur across the segment.

- Anisotropic prefiltering is available on the graphics pipeline for anisotropic sampling in arbitrary directions, addressing the issue of unwanted blurring across the segment direction. This approach is appealing but has the following downsides: 1) the amount of anisotropy is hardware-limited, yielding

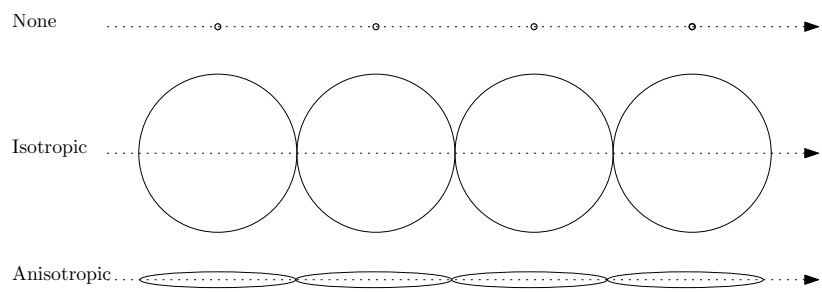

Figure 2: Prefiltering options for subsampling 1D integrals

only a linear reduction on the number of texture samples, as in the previous two options. 2) The combination of the hardware-provided anisotropic filter kernels only provide an approximation of the desired linear integral.

These approaches do not feature the same factor but all have nonetheless a linear time-complexity relative to the length of the projected segment. By restricting ourselves to linear box filtering, we demonstrate how the epipolar nature of the filtering geometry (section 2.1) may be used to implement box-filtered blending in constant-time (section 2.3).

\subsection{Constant-Time Box-Filtered Blending with Integral Ra- dial Images}

We introduce the integral radial image at a given pole as a prepocessed image that enables the computation of $1 \mathrm{D}$ box filters in constant time, provided that the supporting line of the box filter passes through the pole of the integral radial image. The computation of the integral radial image of a image at a given pole is performed as follows:

1. Resample the image in radial geometry $x_{i j}$ with the desired pole. Details follow in section 2.4.

2. Compute the Integral Radial Image by computing partial vertical sums of the radial image : $X_{i j}=\sum_{k=1}^{j} x_{i k}$

1D radial box filters correspond to $1 \mathrm{D}$ vertical box filters in a radial resampling of the input image. Since the preprocessed integral radial image stores vertical partial sums, only two texture lookups on the integral radial image are required:

$$
\sum_{k=j_{0}}^{j_{1}} x_{i k}=\sum_{k=1}^{j_{1}} x_{i k}-\sum_{k=1}^{j_{0}-1} x_{i k}=X_{i, j_{1}}-X_{i, j_{0}-1}
$$

Thus, 1D radial box filters may be performed in constant time on the integral radial image.

\subsection{Implementation details}

Integral Radial Images are 1D integral images similar to the well known 2D integral images or Summed Area Tables. When implementing $1 \mathrm{D}$ or $2 \mathrm{D}$ integral images, there is a number of technical points that need attention (Hensley et al., 2005):

Partial sum complexity The sequential computation of running (i.e. partial) sums is linear in the size of the image $(W, H)$. Independent computation of each partial sum is however suboptimal in a parallel algorithm. In the parallel context of an OpenGL implementation, they are best implemented as $O(\log H)$ fragment shader passes. 
Precision To prevent artefacts, precision loss and type overflow issues must be addressed. Integral Radial Image are 1D rather than 2D-integral images, which reduces the exact computation requirements are the running sums are over a linear rather than areal number of image samples. Integer-typed textures must nonetheless be used with the sufficient number of channels: $N+$ $\left\lceil\log _{2}(H)\right\rceil$ bits for exact handling of $N$-bit textures.

Radial Resampling Geometry This aspect is specific to Integral Radial Images and is one of the contributions of this paper. The parametrization of the radial geometry must be performed with a number of criteria in mind. It must map to a regular square grid to simplify both addressing and interpolation code and enable hardware support. Furthermore, we would like it to be as lossless as possible by maintaining the full resolution everywhere while limiting the extra space due to the mapping of the point location of the pole to a linear location in the radial parametrization. We propose to decompose the radial resampling function in 2, 3 or 4 patches depending on the position of the (possibly infinite) pole (figure 3 ). More precisely, the projective texture image domain may be seen as the intersection of 4 half-spaces which boundary lines support the 4 edges of the image domain. We create a triangular patch in texture space for each such half-space that contains the pole. This patch has a triangular shape with a vertex at the pole and the opposite segment at one of the 4 image edges. To prevent undersampling, we distribute as many pixels along the edge in the patch as in the image edge and as many pixels vertically as the pixel distance between the pole and the line supporting the image edge. This simple parametrization is rather intuitive and easier to handle than a spherical parametrization. Furthermore it has the advantage of at most doubling the storage requirements. Furthermore, it is piecewise projective and thus well supported by the graphics hardware.

\subsection{Linear Graph Navigation}

The Integral Radial Image preprocessing has to be performed for each image as soon as the projection of the virtual viewpoint changes on the projective image. There are however cases where this change is rare. For instance if the viewpoint is moving on the straight line joining two projective texture centers, then its projections in each texture are constant and thus the preprocessing may only be performed once. By extension, the same argument applies when navigating in a graph, which vertices are the projective texture centers and which linear edges encode allowable paths between the two projective textures that are to be blended.

\section{UNCERTAIN GEOMETRY}

We introduce the following uncertain scenes of increasing complexity which may be used in a projective texturing context.

\subsection{Geometry-less Filtered Blending}

In this case, the only piece of 3D information used is the poses and calibrations of the projective textures and a very rough prior on the scene geometry. Namely, we assume that we have an oracle function that gives, for each pixel of the interpolated view, the minimum and maximum scene depths $\left[d_{\text {near }}, d_{\text {far }}\right]$. A simple scene-agnostic prior might be that there is an unoccupied sphere of radius $r$ around the virtual viewpoint, such that the uncertain depth interval is $[r,+\infty]$. If $r$ is not too small compared to the distance between the projective texture center and the virtual viewpoint center, this will yield an uncertain projected segment of reasonable extent.

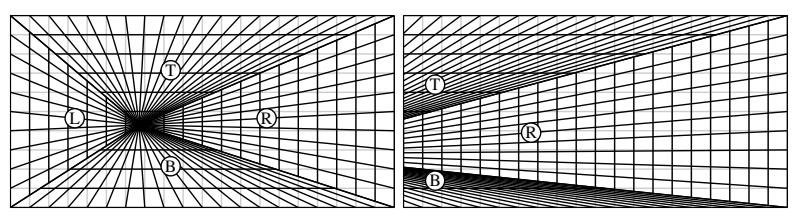

(a) Original images with a pole inside (left) and outside (right) the image domain. Up to 4 resampling grids are used to perform the piecewise-projective radial image resampling : Top, Bottom, Left and Right.
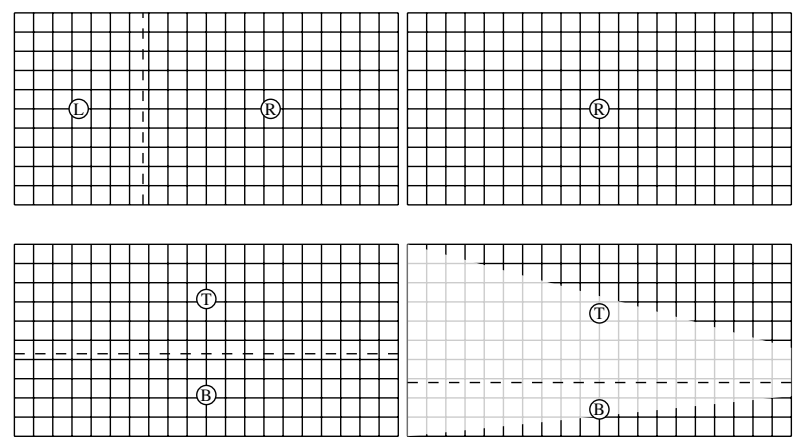

(b) Resampled image pairs Top/Bottom and Left/Right (left). The pole being placed on the left of the image domain, the Left resampling grid is not used and the Right resampling grid occupies the full image grid. Furthermore, less than half of the Top and Bottom pixels are sampled from the image domain, resulting in some memory over-allocation (right).

Figure 3: Resampling Geometry of the Radial Images. Pixel centers correspond to grid line crossings.

This may be implemented in OpenGL with a screen-aligned quad coupled with a simple fragment that implements the oracle depth interval function based on the screen pixel coordinates and the view geometry. The depth interval is unprojected from the view geometry to a 3D line segment, which is then reprojected to the projective textures. If box filtering is to be achieved, then 2 lookups to the Integral Radial Images per screen pixel per projective texture are sufficient, yielding a very efficient implementation of filtered blending which performance is independent of the scene complexity and the virtual viewpoint location.

\subsection{Filtered Blending}

Among the 3 dimensions of space, 1 dimension of the geometric uncertainty may easily be tackled. This is the geometric uncertainty along the rays between the scene points and the virtual viewpoints. This corresponds to a depth uncertainty perceived from the virtual viewpoint, with no questioning on the scene silhouettes.

This may be achieved by using the regular graphics pipeline to compute the usual Z-buffer (depth image $z(i, j)$ ), which produces the depth interval $[z(i, j)-\delta, z(i, j)+\delta]$, where $\delta$ is depth uncertainty parameter. This parameter may be an overall constant or an interpolated attribute on the geometry. This depth interval may directly be used as the depth oracle of the previous section. This corresponds to the original uncertain scene handling of (Eisemann et al., 2007).

\subsection{Silhouette-aware Filtered Blending}

As an extension to the previous section, we would like to address the 3 dimensions of depth uncertainty. Namely we would like to introduce silhouette uncertainty as well as depth uncertainty. 
Here, we propose a general set-up where the scene surface is given by two bounding surfaces, the near and far surfaces, which may be arbitrarily complex, but yet fulfil the following requirements :

1. The near, far and scene surfaces are all watertight, oriented and not self-intersecting. Thus, they properly define a partition of space into interior and exterior volumes. Two such surfaces have an inclusion relationship if the interior volume of the former is strictly contained in the interior volume of the latter.

2. Inclusion. The far surface is included in the scene surface which is in turn included in the near surface, effectively bounding the uncertain scene by these two accessory surfaces.

In probabilistic terms, these bounding surfaces provide the support of a stochastically distributed uncertain scene surface. This provides, for each viewing ray originating outside the far surface the set of possibly many depth intervals which contain the intersection of the ray with the scene. Namely, the scene intersection is a point between the viewpoint and the first intersection of the far surface along the viewing ray which is inside the near surface. Please notice the different roles of the near and far surfaces. The far surface acts as an opaque surface that blocks the view ray, while the interior of the near surface acts as a transparent volume that the viewing ray may enter or exit (figure 4).

Based on this set-up, what is needed to use the method developed in the previous sections is the probability of the first intersection of the scene along the viewing ray (assuming an opaque scene surface). This will enable specifying the synthetic view color as the expectation of the color of this stochastic intersection point.

In this work, we propose to use the most straightforward probabilization, namely a uniform probability on the projection on the sampled texture of the multi-interval support defined by the intersection of the viewing ray with the bounding surfaces, leaving more complex probabilizations for future work.

The computation of the expected color based on this uniform probability may be carried out very efficiently on the GPU, provided that Integral Radial Images are available. Let us denote $e$ the epipole and $s \mapsto p(s)$ a parametrization of the projection of the viewing ray on the projective texture. Then, the segments $\left[p\left(s_{i}\right), p\left(s_{i}^{\prime}\right)\right]_{i=1 \ldots N}$ are defined as the colinear segments in texture coordinates of the projections the line segments resulting from the intersection of the viewing ray with the uncertain surface. Note that $e$ may be equal to $p\left(s_{1}\right)$ (when $s_{1}=0$ ) if the virtual viewpoint is inside the near surface and that all points $p\left(s_{i}\right)$ and $p\left(s_{i}^{\prime}\right)$ are projected intersections of the ray with the near surface except $p_{N}^{\prime}$ which is the projection of the first intersection of the ray with the far surface. Denoting $c(\cdot)$ the color-texture lookup function, the expected color is then given by :

$$
\begin{aligned}
{\left[s_{i}, s_{i}^{\prime}\right]_{i=1 \ldots N} } & \mapsto \frac{\sum_{i} \int_{s_{i}}^{s_{i}^{\prime}} c(p(\cdot))}{\sum_{i} \int_{s_{i}}^{s_{i}^{\prime}} 1} \\
& =\frac{\sum_{i} \int_{0}^{s_{i}^{\prime}} c(p(\cdot))-\int_{0}^{s_{i}} c(p(\cdot))}{\sum_{i} s_{i}^{\prime}-s_{i}}
\end{aligned}
$$

This clearly shows that this value may be computed by accumulating the homogeneous quantities $\pm\left(\sum_{i} \int_{0}^{s} c(p(\cdot)), s\right)$ to get a 4-channel image $\left(\sum_{i} \int_{s_{i}}^{s_{i}^{\prime}} c(p(\cdot)), \sum_{i} s_{i}^{\prime}-s_{i}\right)$, which may be

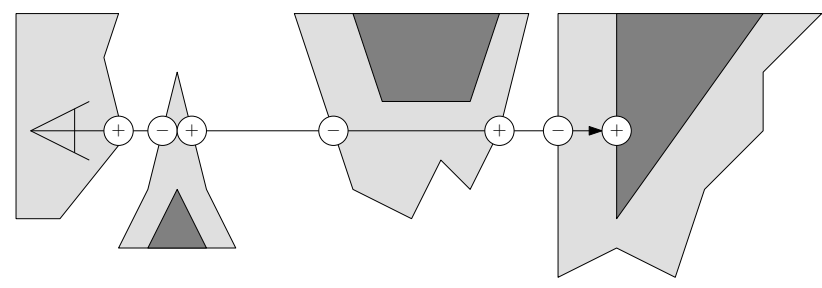

Figure 4: The eye ray possibly exits (+) and reenters (-) the interior of the near surface (light gray) multiple times before hitting the far surface (dark gray).

\begin{tabular}{|c|c|c|c|c|}
\hline P & Surf. & Face & Mode & Value \\
\hline 1 & Far & Front & Replace & $s_{N}^{\prime} \mapsto\left(+\int_{0}^{s^{\prime}}{ }_{N} c(p(\cdot)),+s_{N}^{\prime}\right)$ \\
\hline 2 & Near & Front & Add & $s_{i} \mapsto\left(-\int_{0}^{s_{i}} c(p(\cdot)),-s_{i}\right)$ \\
2 & Near & Back & Add & $s_{i}^{\prime} \mapsto\left(+\int_{0}^{s_{i}^{\prime}} c(p(\cdot)),+s_{i}^{\prime}\right)$ \\
\hline 3 & Quad & Front & Replace & $(r, g, b, a) \mapsto \frac{(r, g, b)}{a}$ \\
\hline
\end{tabular}

Table 1: Summary of the 3 draw passes P1, P2 and P3 used to render an uncertain scene bounded by near and far surfaces, under a uniform probability on the projected points on the sampled texture.

post-processed in a final pass by dividing this quantity by its last component.

In OpenGL terms, it amounts to drawing first the far surface with the usual settings (depth test enabled, back face culling, blending mode set to replace) followed by a subsequent draw call with the near surface geometry with depth test enabled to get the occlusion of the far surface but depth write disabled, alpha test disabled to prevent the filtering negative alpha values and a simple additive blending mode. The sampled values are looked up using a single texture sample on the Integral Radial Image and blended (in replace, add or subtract modes depending on the surface type and orientation according to table 1) into a 0 -initialized floating point offscreen color buffer. The last pass is a simple screen-aligned quad which performs the final division in the fragment shader.

This proposed rendering pipeline enable the visualization of uncertain silhouettes, extending the uncertain-depth-only filtered blending. The proposed probabilization is the one that yields the simplest and most efficient implementation. As the subsequent intervals are weighted by their respective lengths, and there is no transparency support, the rendering is order independent, and thus there is no need to depth-sort the surfaces.

The rendering however is however limited to occlusions cast by the far surface. Occlusion is thus not fully supported: it does not handle self occlusion by the uncertain scene surface and occlusion between the scene surface and the projective texture center. This would however likely require the adaptation of transparency handling methods such as depth sorting or depth peeling.

\section{RESULTS}

\subsection{Geometry-less Filtered Blending}

Such values may be given for instance by the assumption that the scene is higher than a flat plane modeling the ground and a vertical circular paraboloid centered around the interpolated viewpoint (figure 5). Figure 6 show screenshots of the accompanying video that showcases the resulting interpolated video from a set of 12 georeferenced calibrated images taken from a mobile mapping system every 3 meters. 
near paraboloid

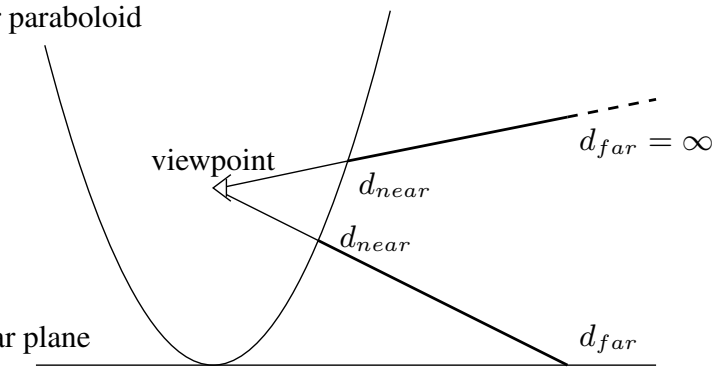

Figure 5: Uncertain scene bounded by a near paraboloid and a far plane.

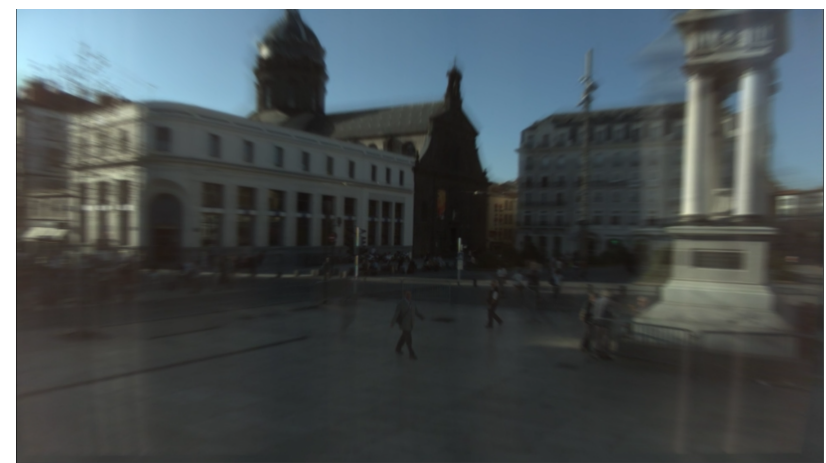

Figure 6: Screenshot of the accompanying video sequence.

The scene prior is given by the geometry of the mobile mapping system : the far plane is defined by slightly lowering (by $10 \mathrm{~cm}$ to get some margin) a guess of the plane of contact of the vehicle wheels and the circular paraboloid of the near plane is defined by the viewpoint location, the far plane and a radius of 10 meters at the level of the interpolated viewpoint.

The virtual viewpoint of figure 6 is generated in between two consecutive views at two thirds of the line segments joining one neighboring view to the other. The result of the projective texturing of these two views have been blended, as one is fading in and the other is fading out.

Of particular interest are the following properties : the pixels corresponding to the direction of the motion suffer limited or no blur. Pixels away from this direction exhibit only a 1D radial blur, similar to the Star Trek-like "hyperspace" effect. Distant and ground objects feature no aliasing, thanks to the epipolar radial blur of sufficient extent. The depth of the near surface has however been underestimated on the right of the scene, resulting in some limited ghosting of the monument. Decreasing the depth of the near surface enough to make it nearer than the scene at every pixel would have extended the radial blur of the pedestal in the two textures up to make their blur extent overlapping, trading out the disturbing double image or popping artefact for an increased amount of radial blur.

\subsection{Filtered Blending}

3D city models of ever increasing accuracy are produced and maintained worldwide. To illustrate our method, we use a coarse 3D city model of metric accuracy (corresponding to CityGML LOD1), which is the BDTOPOC, produced nationwide by IGN, the French National Mapping Agency. The scene is composed of flat-roof buildings together with a metric accuracy Digital Terrain Model (Ground Height map). Figure 7 shows results when varying the constant overall depth uncertainty parameter. A depth un-
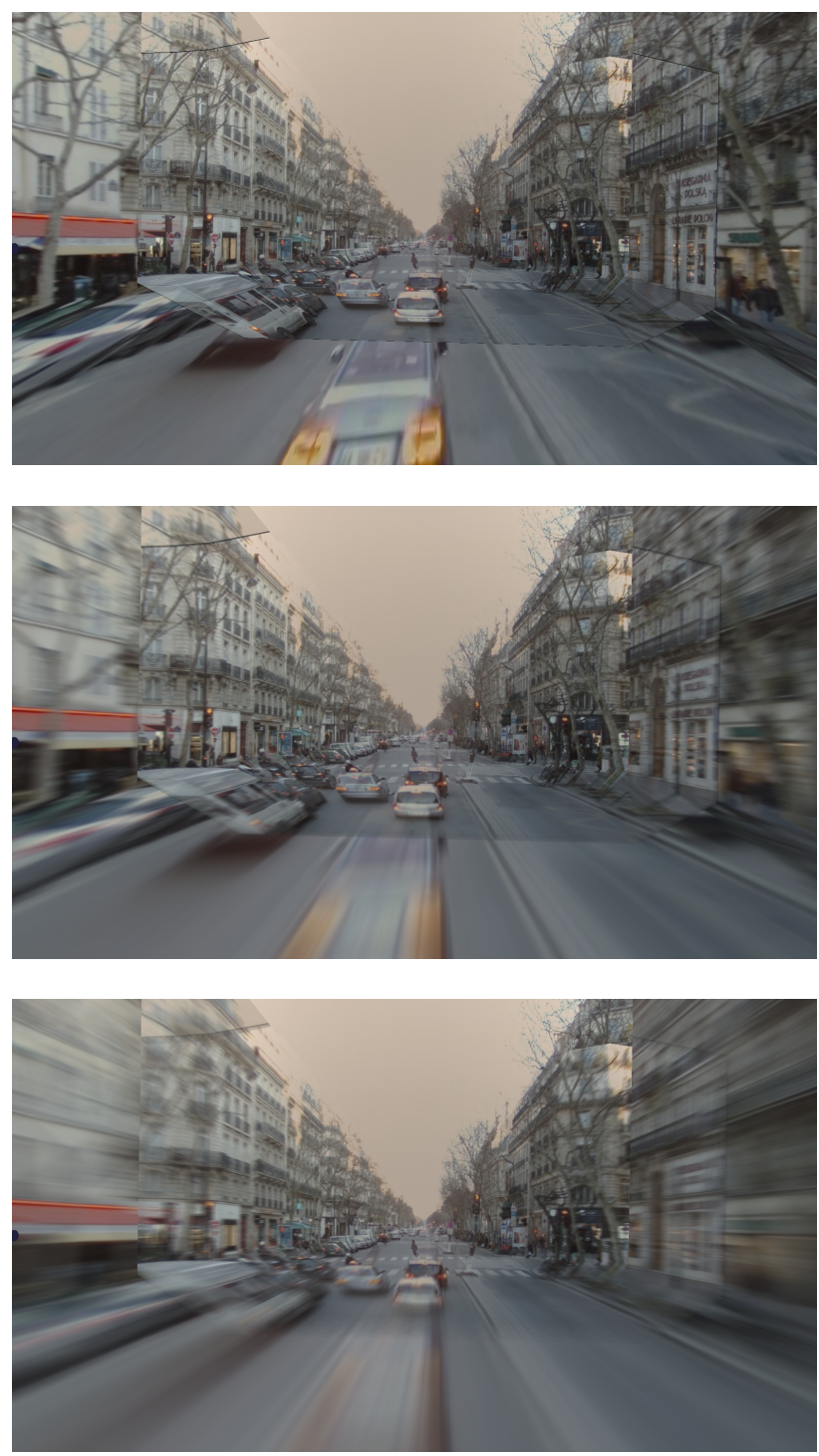

Figure 7: Regular filtered blending. (Top) No uncertainty has been introduced: this is only a linear blend of two 16-meterseparated textures projected on a LOD1 city model proxy. (Middle) 1 meter depth uncertainty. (Bottom) 4 meters depth uncertainty.

certainty of 4 meters is admittedly exaggerated but shows clearly the effect of such a large depth uncertainty. Scene geometry uncertainty is only considered in the depth direction but not in the 2 others. The shortcomings of this depth-only approach is twofold : surfaces seen at grazing angles are affected by a uncertainty that is almost coplanar with the surface, such that the uncertainty effect is underestimated. This could be prevented by taking into account the normal orientation of each pixel to compensate this underestimation. This would however only emphasize the second caveat : silhouettes remain sharp and introduce noticeable artefacts that are addressed in the following section.

\subsection{Silhouette-aware Filtered Blending}

Near and far surfaces may be extracted from the metric-accuracy scene of the last section using the following procedure :

1. Merge all building geometries, the ground mesh and a hollow distant cube modelling the sky to get a single unified triangular mesh, using the union set operator. 
2. Compute a displacement vector for each vertex of the mesh based on the normals of its neighboring triangles.

3. Add (resp. subtract) this vertex displacement attribute to the vertex locations with an overall scaling to yield the near (resp. far) surface at a given level of uncertainty.

Such a displacement vector may be defined as the average of the set of unique normal values of the adjacent triangles, taking the vertical direction for the triangles originating from the DTM, the roofs and the top of the skybox and the horizontal facade normal for the building facades and the skybox sides. This choice provides a $2.5 \mathrm{D}$ extension of the $2 \mathrm{D}$ mittered polygons provided by the straight skeleton (Aichholzer et al., 1995) of the building facades. Please note that step 3 does only produce valid near/far surfaces (watertight, orientable, not self intersecting with the inclusion property) for a limited range of scaling of the displacement field. Arbitrary uncertainty scaling may only be performed correctly using more complex computational geometry algorithms (de Berg et al., 1997) such as a 3D Minkowski sum and differences or a full-blown 2.5D extension of straight skeleton algorithm that would handle topological events, conceptually performing the straight skeleton on each level set of the $2.5 \mathrm{D}$ mesh.

The input 3D city model used for this study used the 3D Minkowski sum approach (figure 8). Figure 9 provides a visualization of this uncertain 3D city model by displaying the normalizing factor $\sum_{i} s_{i}^{\prime}-s_{i}$. The halos around the buildings clearly show the regions of uncertainty and how depth-uncertainty grows at grazing angles.

Figure 10 shows how the silhouette are made uncertain by the proposed approach. A primary remark is that whereas integral radial images were only necessary for large radial blur extents, the proposed silhouette-aware extension manipulates directly its partial sums : without a precomputation technique like integral radial images, partial sums from the would have to be recomputed from scratch for every pixel over possibly long $2 \mathrm{D}$ line segments even for small resulting uncertainty blur. For instance the framerate drops from $60+$ (caped) frame per second to 5 when integral radial images are disabled on our current implementation on a NVidia GPU GTX Titan.

The hard edge of the silhouette is however not fully removed. While the silhouette of the near surface produces a continuous (but not $C^{1}$ ) result, the silhouette of the far surface still produces a discontinuous edge. Even if it is of smaller magnitude than the silhouette-unaware discontinuity, it may still be disturbing. This effect is due to our simple uniform projection density model, which causes the normalizing factor to be discontinuous as the viewing ray crosses past a far surface silhouette : this disoccludes new portions of the viewing ray which are taken into account and thus changes discontinuously the normalization factor.

\section{CONCLUSIONS AND PERSPECTIVES}

This paper proposed successful extensions to the filtered blending approach that provide a continuum of approaches between the geometry less set-up with a loose scene depth prior to a general set-up of an uncertain scene confined in the volume bounded by two arbitrary surfaces.

Integral radial image may prove to be of independent interest in applications that perform radial box filtering of sufficiently many pixels with the same pole. Integral radial images allowed the efficient implementation of a silhouette-aware projective-texturing
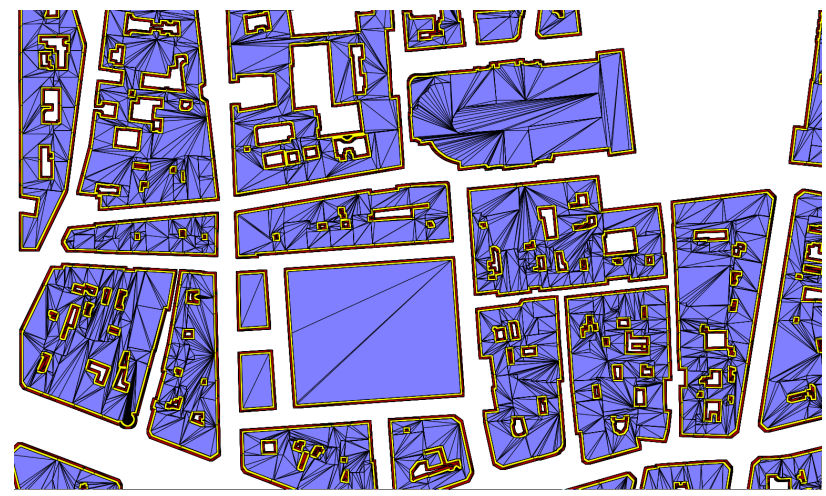

Figure 8: GIS view of the uncertain 3D scene obtained by the minkowski sum (red) and difference (blue) of the original 2.5D building database (yellow). The near and far ground surfaces (MNT) are not shown here for clarity.

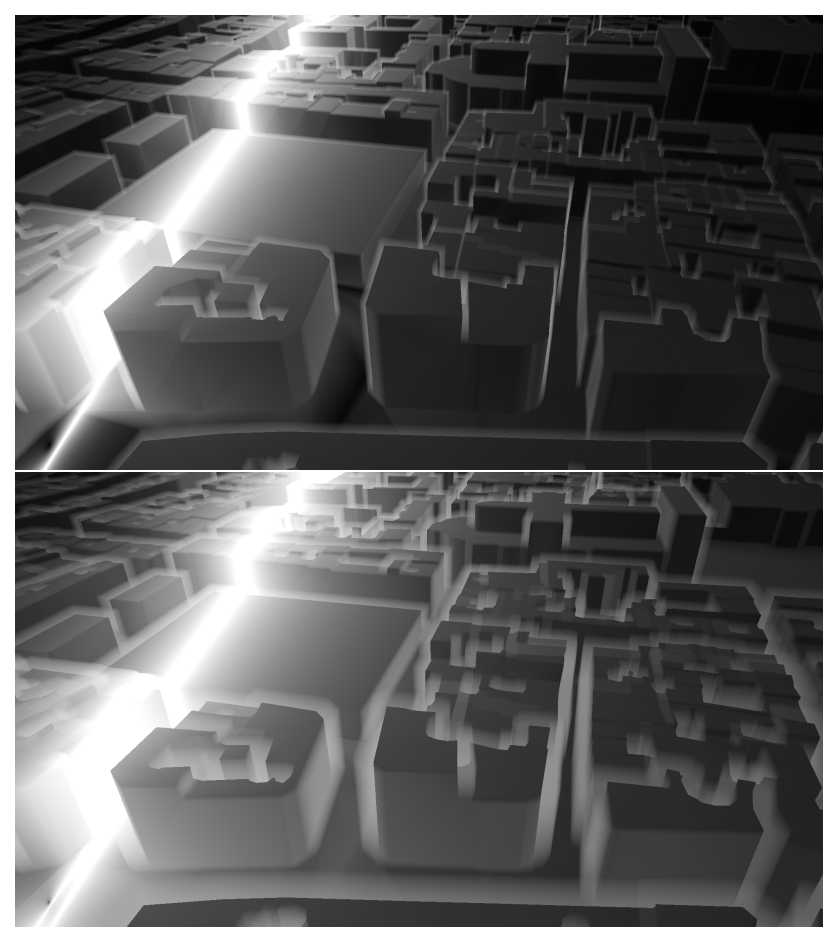

Figure 9: Aerial view of the uncertain model with differing structuring element sizes. Brightness corresponds to the length of intersection of the viewing ray with the uncertain scene, measured in pixels in projective texture space. Thus, this is exactly the content of alpha channel before the final post-processing pass. The white stripe on the left corresponds to the projective image plane, where depth uncertainty has maximal effect. The black spot on the lower left corresponds to the direction of the projective image center, which is sharp no matter the scene uncertainty. 

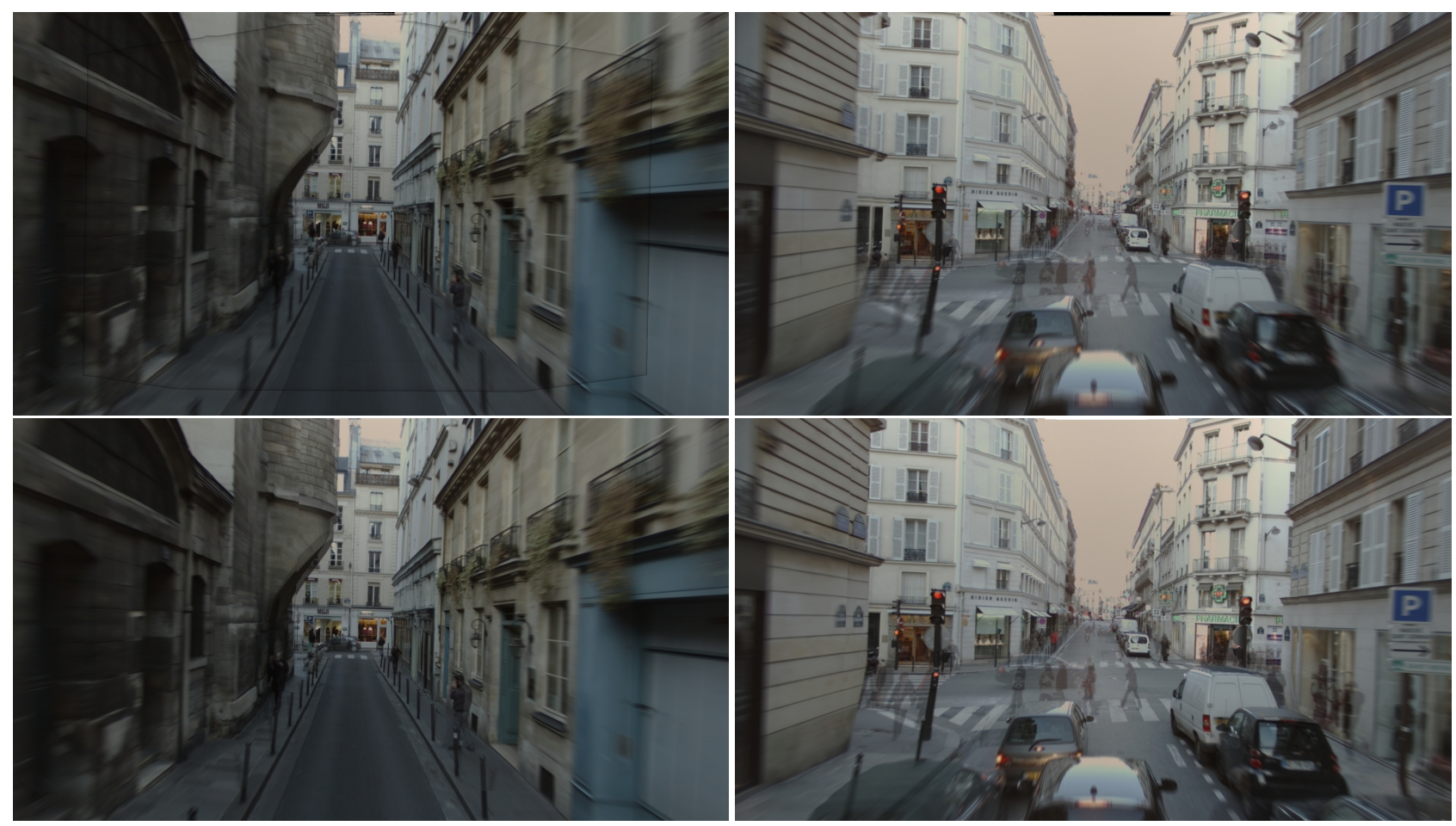

Figure 10: Silhouette-aware filtered blending. (Left) presents a failure case where the uncertain volume is underestimated. The protruding element is not part of the 3D model, which leads to a hard edge in the depth-uncertainty only rendering (top). The proposed approach (bottom) handles the bluring of the pixels up to silhouette of the near surfaces, resulting in a discontinuity artefact. (Right) presents a blending of the 3 nearest views with (top) depth-only uncertainty (+-1m) and with (bottom) silhouette-aware uncertainty (with the cubic structuring element $[-1,1]^{3}$ ). Both views show similar results like the ghosting of mobile objects (pedestrians). The amount of blur in (bottom) is fully 3D and thus takes the surface normal angle into account, which produces more blur on the surfaces that are not front facing(e.g. the ground). The main difference is on the handling of occlusion boundaries (edge of the left building) which trades out the doubling artefact for a blur.

algorithm for uncertain geometry under the specific uniform projection assumption.

Future work on uncertain scenes will include rendering the lidar point cloud with its measurement uncertainties and a diffusion of the depth as an uncertain interval for pixels in-between projected lidar points.

While the assumptions that led to an order-independent rendering method with simple box-filtering enabled high performance rendering, we would like to explore the handling of the various types of occlusion and more elaborated probabilization, so that the resulting image would exactly be the probabilistic expectation of the image when looking at an uncertain scene described stochastically.

\section{ACKNOWLEDGEMENTS}

This material is based upon work supported by ANR, the French National Research Agency, within the iSpace\&Time project, under Grant ANR-10-CORD-023.

\section{References}

Aichholzer, O., Aurenhammer, F., Alberts, D. and Gärtner, B., 1995. A novel type of skeleton for polygons. Journal of Universal Computer Science 1(12), pp. 752-761.
Brédif, M., 2013. Image-based rendering of lod1 3d city models for traffic-augmented immersive street-view navigation. ISPRS Annals of Photogrammetry, Remote Sensing and Spatial Information Sciences II-3/W3, pp. 7-11.

de Berg, M., van Kreveld, M., Overmars, M. and Schwarzkopf, O., 1997. Computational Geometry: Algorithms and Applications. Computational Geometry: Algorithms and Applications, Berlin.

Eisemann, M., De Decker, B., Magnor, M., Bekaert, P., de Aguiar, E., Ahmed, N., Theobalt, C. and Sellent, A., 2008. Floating textures. Computer Graphics Forum (Proc. of Eurographics) 27(2), pp. 409-418. Received the Best Student Paper Award at Eurographics 2008.

Eisemann, M., Sellent, A. and Magnor, M., 2007. Filtered blending: A new, minimal reconstruction filter for ghosting-free projective texturing with multiple images. In: Proc. Vision, Modeling and Visualization (VMV) 2007, pp. 119-126.

Goesele, M., Ackermann, J., Fuhrmann, S., Haubold, C. and Klowsky, R., 2010. Ambient point clouds for view interpolation. Transactions on Graphics 29(4), pp. 95:1-95.

Hensley, J., Scheuermann, T., Coombe, G., Singh, M. and Lastra, A., 2005. Fast summed-area table generation and its applications. Comput. Graph. Forum 24(3), pp. 547-555.

Hofsetz, C., 2003. Image-Based Rendering of Range Data with Depth Uncertainty. PhD thesis, Center for Image Processing and Integrated Computing, University of California, Davis. 